Bio - grafía. Escritos sobre la Biología y su Enseñanza. ISSN 2027-1034

Edición Extraordinaria. p.p. 257 - 264

Memorias del IX Encuentro Nacional de Experiencias en Enseñanza de la Biología y la

Educación Ambiental. IV Congreso Nacional de Investigación en Enseñanza de la Biología.

\title{
MI EXPERIENCIA COMO MAESTRA EN FORMACIÓN EN LA CONSTRUCCIÓN Y APLICACIÓN DE UNA UNIDAD DIDÁCTICA
}

\author{
Daniela Peláez Cárdenas ${ }^{1}$
}

\section{RESUMEN}

Esta experiencia consiste en el diseño y desarrollo de una unidad didáctica sobre el sistema nervioso con estudiantes del grado octavo de mi práctica pedagógica, en una institución rural ubicada en el corregimiento la Floresta del municipio de Maceo (Antioquia). Desde la perspectiva del modelo constructivista, donde los estudiantes son protagonistas de su propio aprendizaje y el rol del docente es el de creador de ambientes de aprendizaje. Con este documento se busca compartir mi experiencia en el diseño y aplicación de una unidad didáctica y los resultados e impactos generados tanto en mis estudiantes como en mi formación como maestra. Además, se pretende que los estudiantes permanezcan activos durante las clases y participen de actividades donde incluyan la escritura y comprensión de textos, el análisis crítico y la argumentación, para que logren entender las funciones del sistema nervioso y su relación con situaciones de su vida cotidiana. La metodología utilizada en el diseño de la unidad didáctica se fundamentó en las cuatro fases del ciclo de aprendizaje de Sanmartí (2005). Inicialmente se me presentaron dificultades en la elaboración de la unidad didáctica, pero, finalmente el impase fue solucionado a partir de la lectura realizada de diferentes documentos. La ejecución de las actividades planeadas tuvo un impacto positivo, ya que los estudiantes se apropiaron de los conceptos y se evidenció un mayor aprendizaje, cambio en sus actitudes y motivación hacia la clase al comparar las ideas previas con el seguimiento y evaluación final. Como ventaja de las unidades didácticas, resalto el trabajo desde diferentes actividades y mayor comprensión de temas complejos como el sistema nervioso. Finalmente, como maestra en formación aprendíla importancia de la planeación de actividades y desarrollo de estrategias orientadas a fortalecer los diferentes estilos de aprendizaje de los estudiantes. Asimismo, desarrollé las clases dejando de lado la idea de que el maestro es el que tiene el conocimiento y otorgándole mayor importancia a las capacidades y producciones de los estudiantes.

PALABRAS CLAVE: Unidad didáctica, práctica pedagógica, estrategias didácticas, desarrollo de competencias, reflexión pedagógica, enseñanza del sistema nervioso.

\footnotetext{
${ }^{1}$ Estudiante de Licenciatura en Educación Básica con énfasis en Ciencias Naturales y Educación Ambiental. Universidad de Antioquia. Sede Magdalena Medio, Puerto Berrío.
} 
Bio - grafía. Escritos sobre la Biología y su Enseñanza. ISSN 2027-1034

\section{Edición Extraordinaria. p.p. 257 - 264}

Memorias del IX Encuentro Nacional de Experiencias en Enseñanza de la Biología y la

Educación Ambiental. IV Congreso Nacional de Investigación en Enseñanza de la Biología.

\section{INTRODUCCIÓN}

Como maestros, es necesario que realicemos una planeación estructurada sobre el tema que se desea abordar y las competencias a fortalecer en clase. Realizar la planeación puede ser un poco tedioso puesto que requiere de tiempo, dedicación y creatividad, sin embargo, es fundamental que cada maestro se prepare bien antes de cada clase, pues considero que sería un acto irresponsable llegar al aula a improvisar. Los estudiantes siempre están atentos a cada detalle y se dan cuenta si su maestro está improvisando o por el contrario ha preparado cada momento de la clase.

En esta planeación no debemos desconocer los estilos de aprendizaje de los estudiantes, es decir, las actividades con las que ellos aprenden mejor, puesto que en un aula de clases tenemos diversidad de estudiantes que aprenden de manera diferente. Durante el tiempo que llevo como maestra en formación, me he dado cuenta que a los estudiantes les aburre la monotonía y la rutina, contrario a esto, les encanta la variedad de actividades donde puedan estar activos e interactuando con los demás compañeros. Esto se ha evidenciado en muchas de las clases donde mis estudiantes han estado un poco distraídos debido a que no se sienten motivados con algunas de las estrategias propuestas en clase.

Por lo tanto, considero que ahora que me estoy formando como maestra debo empezar a cambiar algunas estrategias de enseñanza y a su vez implementar algunas nuevas, pero siempre pensando en los intereses, necesidades y el contexto de los estudiantes. Surge entonces, la necesidad de diseñar una unidad didáctica que me permita por un lado, tener una planeación organizada con tiempo y por el otro, desarrollar diferentes estrategias que estén relacionadas con las necesidades e intereses de los estudiantes. Buscando que ellos sean los protagonistas de la clase, se involucren y puedan construir sus propios conocimientos; también para que desarrollen otras competencias asociadas a la lectura y la escritura.

La unidad didáctica ha sido diseñada desde el modelo constructivista, el cual se caracteriza porque el papel del estudiante siempre es activo, él es quien debe construir conocimientos por sí mismo y extraer sus propios significados (Hernández, 2008). La idea de implementar dicho modelo, surge a partir de mis reflexiones al notar que mis clases han sido muy tradicionales y los estudiantes se estaban quedando en un segundo plano. Con esto no quiero decir que el modelo tradicionalista sea malo, pues a veces es necesario retomar algunas estrategias de éste, pero es fundamental permitir que el estudiante construya, encuentre respuestas, se equivoque y aprenda. Finalmente, lo que pretendo es modificar esas prácticas tan aburridoras para mis estudiantes por otras que los motiven y que a su vez, me ayuden a crecer en este proceso de formación como maestra.

La Unidad didáctica fue elaborada para abordar el tema del sistema nervioso con estudiantes del grado octavo, de una institución rural ubicada en el corregimiento la Floresta del municipio de Maceo (Antioquia). Este grupo es excelente académicamente, 


\title{
Bio - grafía. Escritos sobre la Biología y su Enseñanza. ISSN 2027-1034
}

\section{Edición Extraordinaria. p.p. 257 - 264}

\author{
Memorias del IX Encuentro Nacional de Experiencias en Enseñanza de la Biología y la \\ Educación Ambiental. IV Congreso Nacional de Investigación en Enseñanza de la \\ Biología.
}

son participativos, respetuosos y de buen comportamiento, además, tienen muchos sueños y deseos de estudiar para cumplir sus proyectos de vida. La mayoría de los estudiantes deben recorrer largos caminos para poder llegar a la institución, algunos incluso deben andar en caballo y luego continuar en bus hasta el colegio. Cuando cae agua son varios los estudiantes que faltan a clase, porque es difícil salir de las fincas y los estudiantes que logran llegar al colegio, a veces, tienen que ser evacuados de las aulas de clase porque se mojan en los salones del último piso. Pese a esto, se presentan buenas y adecuadas relaciones interpersonales entre los miembros de la institución. Las personas son colaboradoras, solidarias y se percibe un ambiente agradable y de sana convivencia. Los padres de los estudiantes se dedican a desarrollar actividades como la ganadería, la agricultura, la producción de caña y panela, la minería, entre otras; como sustento económico para sus familias. Algunas personas trabajan en fincas y complementan su sustento económico con el trabajo de cultivos o ganado que tienen en sus pequeñas parcelas. Considero que todas las adversidades por las que tienen que pasar estas familias del área rural para poder enviar a sus hijos a la escuela, es lo que los ha motivado a seguir adelante y valorar lo que tienen, esto se ve reflejado en el desempeño académico de los estudiantes y la motivación que muestran en el desarrollo de la jornada escolar.

\section{Conceptualización}

Las unidades didácticas son herramientas muy usadas por los maestros debido a la acogida y efectividad en las aulas. Corrales (2010) define la unidad didáctica (UD) como una planeación que da a conocer los propósitos de enseñanza aprendizaje que se van a trabajar en el aula de clase. Además, que esta planeación se hace con el objetivo de "conocer el qué, quiénes, dónde, cómo y porqué del proceso educativo, dentro de una planificación estructurada del currículum" (p. 2).

Blasco y Mengual (s.f.) definen la unidad didáctica como una unidad de trabajo planeada por los maestros, en la cual tienen en cuenta las particularidades de cada aula y adecúan las estrategias al currículo de la institución. Igualmente, consideran fundamental incluir las ideas previas de los estudiantes para trabajar a partir de ellas. Su objetivo principal según estos autores es "lograr el aprendizaje de los alumnos en relación con un tema determinado" (p. 4). Por otro lado, Sanmartí (2005) establece que "[...] decidir qué se va a enseñar y cómo, es la actividad más importante que llevamos a cabo los enseñantes ya que a través de ella concretamos y ponemos en práctica nuestras ideas e intenciones educativas" (p. 13).

Por lo anterior, considero que a través del diseño de unidades didácticas los maestros demostramos a nuestros estudiantes la importancia de su aprendizaje, igualmente, nos demostramos a nosotros mismos que podemos crecer y transformar nuestra práctica educativa. Debido a que en el momento de ejecución de las actividades contenidas en la unidad didáctica, se presentan más oportunidades para que los estudiantes comprendan un concepto determinado de acuerdo a las actividades que más se acerquen a su modo de aprender. Algo similar plantea Sanmartí (2005) al mencionar que las actividades de la unidad didáctica son "[...] la hipótesis que formulamos los profesores [...] sobre cuál puede ser el mejor itinerario para nuestros alumnos con el objetivo de que aprendan, 
Bio - grafía. Escritos sobre la Biología y su Enseñanza. ISSN 2027-1034

Edición Extraordinaria. p.p. 257 - 264

Memorias del IX Encuentro Nacional de Experiencias en Enseñanza de la Biología y la

Educación Ambiental. IV Congreso Nacional de Investigación en Enseñanza de la Biología.

teniendo en cuenta tanto los contenidos a introducir como las características y diversidad de su alumnado" (p. 35).

\section{Diseño de la unidad didáctica}

Para diseñar esta unidad didáctica tuve en cuenta el ciclo didáctico que plantea Sanmartí (2005), que consta de las siguientes fases: Exploración, Introducción de los nuevos conocimientos, Síntesis y Aplicación. En cada una de estas fases formulé mínimo dos actividades que apuntan a que los estudiantes lean, analicen, argumenten, escriban, inventen, representen y finalmente experimenten y apliquen los conocimientos que hayan podido adquirir durante el desarrollo de las etapas anteriores de la unidad didáctica. En estas actividades se incluye producción de escritos y solución de talleres a partir de videos, creación de cuentos, argumentación, elaboración de carteleras, cuadros comparativos, análisis de situaciones problemas, experimentación, entre otras.

Teniendo en cuenta el ciclo de aprendizaje de Sanmartí, se presenta a continuación un esquema que representa cada una de esas fases con las respectivas actividades que
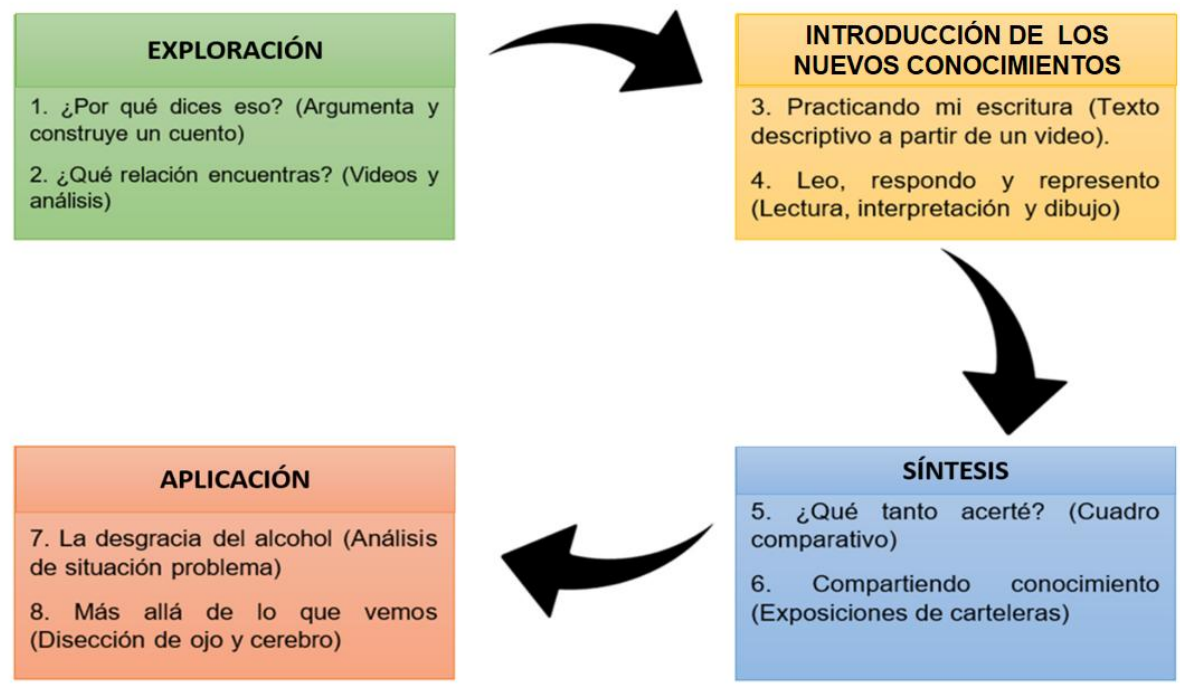

pronı ıce on la ı ınidad didántica (Firı ıra 1)

Figura 1: Fases del ciclo de Sanmartí con las respectivas actividades de la unidad didáctica. Elaboración propia.

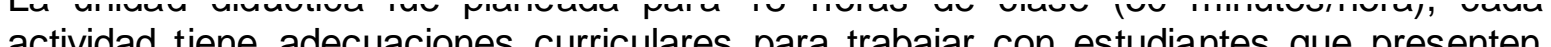
alguna discapacidad, ya sea motora, visual, auditiva, TDAH, entre otras. Asimismo, cada actividad tiene unas normas de clase y la evaluación se hace mediante una rúbrica que valora el desempeño de los estudiantes en cada una de ellas, teniendo en cuenta su participación en clase, el cumplimiento de las normas y de cada una de las tareas asignadas.

Tanto los contenidos como las actividades se diseñaron teniendo en cuenta el contexto donde se encuentran inmersos los estudiantes. Si bien es cierto que en este contexto rural no son tan frecuentes los problemas de drogadicción por sustancias como la 
Bio - grafía. Escritos sobre la Biología y su Enseñanza. ISSN 2027-1034

Edición Extraordinaria. p.p. 257 - 264

Memorias del IX Encuentro Nacional de Experiencias en Enseñanza de la Biología y la

Educación Ambiental. IV Congreso Nacional de Investigación en Enseñanza de la Biología.

marihuana, la cocaína y demás, el alcohol es uno de los enemigos más representativos de estas familias, lo que hace pertinente trabajar el tema en cuestión.

\section{REFLEXIÓN PEDAGÓGICA}

La construcción de la unidad didáctica fue un proceso complejo, tenía claro inicialmente cuáles eran los elementos que debía tener una unidad didáctica, sin embargo, no sabía por dónde empezar. Pero, fueron surgiendo las ideas después de observar diferentes documentos y modelos relacionados con unidades didácticas. En la primera fase de exploración pensé en emplear una estrategia donde implícitamente se pudieran observar conceptos y situaciones donde se viese involucrado el sistema nervioso. Es ahí, donde surge la idea de presentarles algunos videos del chavo del ocho, plantearles afirmaciones de la vida cotidiana y motivarlos a inventar un cuento. Estas actividades se hacen con el fin de observar y conocer, de manera diferente y divertida, qué saben los estudiantes sobre el sistema nervioso.

Posteriormente, empecé a planear las actividades de introducción de los nuevos conocimientos. Fue un proceso aún más difícil, porque tenía la concepción de que el maestro siempre debe explicar para que los estudiantes puedan aprender. Entonces se presentaba una confusión de mis ideas, entre lo que quería hacer desde un modelo constructivista y la concepción que tenía interiorizada de tipo tradicionalista. Sin embargo, luego de varios intentos logré diseñar las actividades y estrategias que se adecuaran al modelo constructivista. Estas actividades están orientadas a que los estudiantes analicen, interpreten y produzcan sus propios textos a partir de un video y lo relacionen con situaciones de su vida cotidiana en el contexto rural. Asimismo, que respondan preguntas donde se evidencie cómo están en comprensión lectora.

En las siguientes fases el proceso fue un poco más fácil, ya estaba encaminándome hacia una forma diferente de diseñar las clases. Surgieron varias actividades que pueden ser motivadoras e innovadoras para los estudiantes. En especial las de aplicación, donde los estudiantes pueden demostrar lo que han aprendido e igualmente hacer algo diferente a lo que se hace en el aula, como por ejemplo las disecciones de ojo y de cerebro.

Finalmente, establecí las normas de cada actividad, las rúbricas de evaluación y las adaptaciones curriculares, basándome en la Guía $N^{\circ} 12$ del Ministerio de Educación sobre la Fundamentación conceptual para la atención en el Servicio educativo a estudiantes con necesidades educativas especiales -NEEDE. Este paso me llevó bastante tiempo, pero no fue tan complejo como el diseño de las actividades. Procedí entonces a ajustar detalles para empezar con la ejecución de la unidad didáctica, la cual está terminada para la ejecución, pero queda abierta a cambios y modificaciones para trabajar con grupos de diferentes contextos o con otros profesores. 


\title{
Bio - grafía. Escritos sobre la Biología y su Enseñanza. ISSN 2027-1034
}

\section{Edición Extraordinaria. p.p. 257 - 264}

\author{
Memorias del IX Encuentro Nacional de Experiencias en Enseñanza de la Biología y la \\ Educación Ambiental. IV Congreso Nacional de Investigación en Enseñanza de la \\ Biología.
}

\section{Puesta en escena de la Unidad Didáctica}

Terminado el diseño de la unidad didáctica, procedí a desarrollarla con mis estudiantes. Al inicio, se sintieron un poco confundidos al socializarles las normas de clase y los criterios de evaluación (leído en sus gestos). Pero, cuando iniciamos las actividades cambió la expresión de sus rostros, su actitud y se involucraron activamente en la clase. Es el caso de un estudiante que trabaja poco y se le llama la atención frecuentemente y desde que estamos realizando dichas actividades, ha tenido un gran cambio, participa en clase, quiere siempre ser el líder y no hay que llamarle la atención.

A parte del cambio en la actitud de los estudiantes, ellos me han expresado algunas de las cosas que han sentido desde que se empezó a desarrollar la unidad didáctica. Algunas de esas expresiones han sido: "La clase estuvo muy buena", "Me gustó mucho la clase porque todos participamos y nos integramos", "Muy divertida la clase y hacemos cosas diferentes mientras aprendemos", entre otras. Todo esto me motiva a seguir aprendiendo como maestra y me ha llevado a reflexionar cada una de mis prácticas educativas, con el fin de estar siempre renovándolas y fortaleciéndolas.

Durante la ejecución de las actividades propuestas, los estudiantes respondieron positivamente a cada una de ellas. En clases anteriores, siempre eran los mismos cuatro o cinco estudiantes que participaban voluntariamente en las actividades de opinión, argumentación, lectura al frente, entre otras. Ahora, voluntariamente participan otros estudiantes y se involucran más en el desarrollo de las diferentes actividades. Se pudo evidenciar también el fortalecimiento del trabajo en equipo y asimismo, el trabajo individual. Pues al inicio, en los trabajos que eran individuales varios estudiantes me entregaban lo mismo. Ahora, cada estudiante sabe que puede pensar por sí mismo y realizar su trabajo a conciencia. Además, expresan sentirse a gusto en las clases y como profesora, evidenciarlo en sus rostros y actitudes es satisfactorio. Por esto, pienso que la ejecución de la unidad didáctica muestra un avance significativo en el proceso de enseñanza y aprendizaje de los estudiantes y de mi proceso como maestra en formación.

Esta unidad didáctica también tuvo un gran cambio en mis conocimientos como profesora de ciencias naturales. Durante las clases me sentía más cómoda, activa, con ganas de seguir formándome como maestra. Por ello, empecé a cambiar mi actitud en el aula, emprendí la búsqueda de nuevas actividades y entendí que no sólo existe un modelo pedagógico: el tradicionalista, sino que existen algunos con los que me siento más identificada, como el constructivista que según Jonassen (citado por Hernández, 2008) "propone que el ambiente de aprendizaje debe sostener múltiples perspectivas o interpretaciones de realidad, construcción de conocimiento, actividades basadas en experiencias ricas en contexto" (p. 27) y que cada uno de estos modelos, puede aportarnos a nuestra labor como docentes.

La actividad llamada "compartiendo conocimiento" fue la que más motivación generó en los estudiantes. En esta actividad, por grupos debían leer algunos artículos sobre los efectos nocivos de las drogas en el sistema nervioso central, posteriormente, elaborar una cartelera para exponerla en otros grupos de la institución invitando a decirle "NO a las drogas". Cuando se inició la elaboración de las carteleras, los estudiantes habían llevado 


\title{
Bio - grafía. Escritos sobre la Biología y su Enseñanza. ISSN 2027-1034
}

\section{Edición Extraordinaria. p.p. 257 - 264}

\author{
Memorias del IX Encuentro Nacional de Experiencias en Enseñanza de la Biología y la \\ Educación Ambiental. IV Congreso Nacional de Investigación en Enseñanza de la \\ Biología.
}

su propio material, frases de concienciación, imágenes alusivas, dibujos entre otros. Los estudiantes manifestaron que sentían nerviosismo, sin embargo, estuvieron muy activos y animados en la realización de las exposiciones, las cuales fueron un éxito porque explicaron las consecuencias del consumo de drogas, se expresaron con claridad y convicción, las carteleras captaban la atención tanto de los estudiantes como de los profesores, debido a los dibujos y las frases motivacionales. Finalmente, los aportes de esta actividad generaron en los demás profesores y compañeros mucha atención y agradecimientos. Esto se evidenció en la actitud de escucha y participación, pues realizaron preguntas y aportes sobre el por qué decir "No a las drogas".

Vivir esta experiencia tan significativa permite contribuir en las mejores prácticas educativas en la institución rural, es así que, compartirla y contarla a otros maestros, especialmente los que también se encuentran en proceso de formación inicial, enriquece nuestra labor. Lo anterior, para dar a conocer otros modos de enseñanza, para motivarlos a que creen sus propias unidades didácticas, que evalúen sus prácticas educativas y las transformen teniendo en cuenta los intereses de sus estudiantes y el contexto en el que se encuentran. Pues la escuela rural tiene dinámicas y situaciones diferentes a la escuela urbana, la escuela rural tiene la capacidad de integrar a toda la comunidad pese a las condiciones de infraestructura, debido a que las familias tienen una valoración positiva de la escuela, aspecto que no es tan evidente en la escuela urbana (Amiguinho, 2011). Asimismo, los maestros en formación debemos tomar riesgos en la práctica pedagógica y proponer a los maestros cooperadores alternativas de enseñanza que involucren al estudiantado y también fortalezcan comunidades de diálogo pedagógico entre maestros.

\section{CONCLUSIONES}

Las actividades y estrategias desarrolladas con el grado octavo han sido muy bien recibidas por los estudiantes. Se notó un avance en ellos en cuanto a la responsabilidad, la escritura, la lectura y la comprensión de textos. Además, su papel ha sido protagónico, pues no es la maestra quien habla y explica siempre, sino los estudiantes quienes están pensando, analizando y produciendo constantemente.

Este proceso no ha sido fácil, ya que la institución es rural y existen pocas herramientas tecnológicas. También, porque los estudiantes vienen acostumbrados a un modelo y ritmo de trabajo diferente, generando dificultad para realizar dichas actividades. Como fortalezas tenemos la perseverancia, las ganas de hacer las cosas bien, la dedicación y la motivación de los estudiantes por salir de la rutina y la monotonía.

Considero que el desarrollo de esta unidad didáctica tiene proyección para generar una transformación en el grado octavo y en la institución. Los estudiantes han perdido el miedo a participar, incluso aquellos estudiantes indisciplinados fueron quienes presentaron mayor participación y se convirtieron en líderes positivos en cada una de las actividades. Ya no hay actitudes negativas ni gestos de pereza, ahora los estudiantes están a la expectativa de cuáles podrán ser las próximas actividades para trabajar en el aula. 
Bio - grafía. Escritos sobre la Biología y su Enseñanza. ISSN 2027-1034

Edición Extraordinaria. p.p. 257 - 264

Memorias del IX Encuentro Nacional de Experiencias en Enseñanza de la Biología y la Educación Ambiental. IV Congreso Nacional de Investigación en Enseñanza de la Biología.

La realización de las unidades didácticas tiene varias ventajas, tanto para el maestro como para los estudiantes. Considero que una de esas ventajas es el tiempo que se ahorra y la tranquilidad que puede adquirir un profesor mientras desarrolla la unidad didáctica que ya tiene estructurada. Además, Sanmartí (2005) menciona que una de esas ventajas puede ser: "motivación para el alumnado, porque encuentran sentido a aquello que aprenden" (p. 29). Igualmente, es algo que juega a favor de los estudiantes, ya que un mismo tema se trabaja desde diferentes actividades y es más probable que los estudiantes logren comprender los conceptos.

\section{REFERENCIAS BIBLIOGRÁFICAS}

Amiguinho, A. (2011). La escuela en el medio rural: educación y desarrollo local. Revista de Currículum y Formación de Profesorado, 15(2), 25 - 37. Recuperado de http://www.ugr.es/ recfpro/rev152ART2.pdf

Blasco, J. y Mengual, A. (s.f.). Las unidades didácticas. Educación Física y su didáctica II. Recuperado de https://rua.ua.es/dspace/bitstream/10045/8092/6/Las\%20unidades\%20b\%C3\%A1sicas\%2 0de\%20programaci\%C3\%B3n.pdf

Corrales, A. (2010). La programación a medio plazo dentro del tercer nivel de concreción: las unidades didácticas. EmásF: revista digital de educación física, (2). Recuperado de https://dialnet.unirioja.es/descarga/articulo/3175435.pdf

Hernández, R. S. (2008). El modelo constructivista con las nuevas tecnologías: aplicado en el proceso de aprendizaje. Revista de Universidad y Sociedad del conocimiento, 5(2). Recuperado de http://www.redalyc.org/articulo.oa?id=78011201008

Sanmartí, N. (2005). La unidad didáctica en el paradigma constructivista. En Couso, D. et al. Unidades didácticas en ciencias y matemáticas. Editorial Magisterio. Capítulo, 1, 13-55. Recuperado de http://ocw.pucv.cl/cursos-1/didactica-i/materiales-de-clases-1/09-launidad-didactica-en-el-paradigma-constructivista 